\title{
Über den Atlas und Epistropheus bei den eingeborenen Formosanern (Seiban).
}

\author{
Von \\ Takamichi Tsusaki. \\ (Aus dem Aratomicchen Instiiut der Medizinischen Hochschule zu Keijo). \\ Mit 6 Figuren auf Tafel XIII.
}

\section{Einleitung.}

Da uns die rassenanatomischen Kenntnisse über die eingeborenen Formoşaner heute noch fast gänzlich fehlen, so beschäftligte ich mich seit Jahren etwas damit und habe schon einige Arbeiten darüber veröffentlicht. Über die Wirbelsäule des Menschen sind im allgemeinen ziemlich genane Untersuchungen schon von vielen Autoren angestellt worden. Der Atlas und der Epistropheus. wurden dabei aber wenig beachtet, besonders sind sie bei den Formosanern vollständig vernachlässigt worden. Die vorliegende Abhandlung über die zwei betreffenden Wirbel der Formosaner zeigt uns, dass zwischen den letzteren und den Japanern in vielen Punkten merkliche Unterschiede bestehen.

\section{Material und Messungsmethode.}

Was das Material anbelangt, so kamen zur Untersuchung 22 Atlanten und 20 Epistrophei in der anatomischen Sammlung zu Taihoku, die alle zu mazerierten Schädeln von erwachsenen Individuen gehören. Aber zu meinem Bedauern waren Geschlecht und Alter des öfteren unklar. Bei der Messungder beiden Wirbel habe ich mich hauptsächlich der Methode von Hasebe (13) angeschlossen und folgende Teile jedes Knochens gemessen :

A. Beim Atlas :

1. Totaler Sagittaldurchmesser, vom Tuberculum anterius bis zum Tuberculum posterius.

2. Totaler Transversaldurchmesser, Entfernung zwischen den äussersten Ausladungen der Processus transversi.

3. Vertikaldurchmesser des Arcus anterior in der Medianlinie. 
4. Vertikaldurchmesser des Arcus posterior.

5. Sagittaldurchmesser des Foramen vertebrale.

6. Grösster Transversaldurchnesser des eigentlichen Foramen vertebrale.

7. Vorderer Transversaldurchmesser des Foramen vertebrale, zwiechen den Winkeln, die durch den Arcus anterior und die Facies articularis inferior gebildet werden.

8. Grösste Weite (Entfernung der äussersten Ausladung) der Facies articulares inferiores.

9. Grösste W'eite (Entferuung der äussersten Ausladung) der Foveae articulares superiores.

B. Beim Epistropheus :

1. Die vordere ganze Höhe, parallel der Achse des Körpers vom Scheitel des Dens bis zum vorderen Rande der unteren Endfläche in der Medianebene.

2. Die hintere ganze Höhe, bis zum hinteren Rand der unteren Endfläche in der Medianebene.

3. Die vordere Höhe des Dens.

4. Die vordere Höhe des Körpers.

5. Die Distanz zwischen den äussersten Ausladungen der Processus transversi (ganze Breite).

6. Der grösste Sagittaldurchmesser des Dens.

7. Der Transversaldurchmesser des Dens in derselben Ebene.

8. Die Distanz zwischen den äussersten Ausladungen der Processus artiçulares superiores.

9. Die Distanz zwischen den äussersten Ausladungen der Processus articulares inferiores.

10. Der Sagittaldurchmesser der unteren Endflächẹ.

11. Der Transversaldurchmesser der unteren Endfläche.

12. Der Sagittaldurchmesser des Foranen vertebrale.

13. Der Transversaldurchmesser desselben.

Ausserdem habe ich noch Messungen an einigen Teilen der beiden Wirbel vorgenommen. 


\section{Eigene Befunde.}

\section{Atlas.}

A. Ergebnisse der Messungen.

1. Totaler Sagittaldurchmesser (Tabelle 1).

Von den totalen Sagittaldurchmessern des Atlas von Formosaneru beträgt, wie die Tabelle 1 zeigt, der grösste $48.0 \mathrm{~mm}$, der kleinste $39.8 \mathrm{~mm}$; durchsehnittlich $44.1 \mathrm{~mm}$. In den meisten Fällen schwankt er zwischen $42.0 \mathrm{~mm}$ und $46.0 \mathrm{~mm}$. Nach Dubreuil-Chambardel (7) misst bei den Europäermännern der grösste totale Sagittaldurchrnesser $51.0 \mathrm{~mm}$, der kleinste $43.0 \mathrm{~mm}$, durchschnittlich $46.5 \mathrm{~mm}$; bei den Weibern der grösste $45.0 \mathrm{~mm}$, der kleinste $43.0 \mathrm{~mm}$,

Tabelle 1.

Totaler Sagittaldurchmesser.

\begin{tabular}{|c|c|}
\hline $\mathrm{mm}$ & Fälle \\
\hline $39.8-40.0$ & 3 \\
$40.1-41.0$ & 0 \\
$41.1-42.0$ & 0 \\
$42.1-43.0$ & 2 \\
$43.1-44.0$ & 6 \\
$44.1-45.0$ & 3 \\
$45.1-46.0$ & 4 \\
$46.1-47.0$ & 0 \\
$47.1-48.0$ & 3 \\
\hline
\end{tabular}

durchschnittlich $43.2 \mathrm{~mm}$. Nach Hasebe (13) beträgt bei den Europäern der grösste $49.0 \mathrm{~mm}$, der kleinste $41.0 \mathrm{~mm}$, durchschnittlich $46.0 \mathrm{~mm}$; bei den Amerikanern durchschnittlich $43.3 \mathrm{~mm}$, während bei den Japanermännern der grösste $47.0 \mathrm{~mm}$, der kleinste $42.0 \mathrm{~mm}$, durchschnittlich $44.5 \mathrm{~mm}$, bei den Weibern der grösste $46.0 \mathrm{~mm}$, der kleinste $39.0 \mathrm{~mm}$, durchschnittlich $41.3 \mathrm{~mm}$ beträgt. Nach Frizzi (11) misst bei den NeuPommern der grösste Durchmesser $47.0 \mathrm{~mm}$, dèr kleinste $42.0 \mathrm{~mm}$, durchschnittlich $44.6 \mathrm{~mm}$. Somit ist der betreffende Durchmesser bei den Formosanern etwas kleiner als bei den Europäermännern, etwas grösser als bei den Europäerweibern, Amerikanern und Japanerweibern, und beinahe gleich gross wie der der Japanermänner und der Neu-Pommern.

2. Totaler Transversaldurchmesser (Tabelle 2).

Der grösste totale Transversaldurchmesser beträgt $78.7 \mathrm{~mm}$, der kleinste $66.5 \mathrm{~mm}$; durchschnittlich $73.0 \mathrm{~mm}$. Grösstenteils schwankt er zwischen 71.0 mm und 76.0 mm. Nach Dubreuil-Cha mbardel (7) misst er bei den Europäermännern $90.0 \mathrm{~mm}$, der kleinste $74.0 \mathrm{~mm}$, durchschnittlich $83.0 \mathrm{~mm}$; bei den Europäerweibern der grösste $76.0 \mathrm{~mm}$, der kleinste $65.0 \mathrm{~mm}$, durchschnittlich 
$72.0 \mathrm{~mm}$. Nach Hasebe (13) beträgt er bei den Europäern $85.0 \mathrm{~mm}$, der kleinste $73.0 \mathrm{~mm}$, durchschnittlich $80.9 \mathrm{~mm}$; bei den Amerikanern durchschnittlich $80.1 \mathrm{~mm}$. Nach demselben Autor misst bei den Japanermännern der grösste $82.0 \mathrm{~mm}$, der kleinste 78.0 $\mathrm{mm}$, durchschnittlich $78.9 \mathrm{~mm}$; bei den Weibern von Japanern der grösste $78.0 \mathrm{~mm}$, der kleinste $65.0 \mathrm{~mm}$, durchschnittlich $71.3 \mathrm{~mm}$. Bei den Neu-Pommern misst er nach Frizzi (11) 77.0 $\mathrm{mm}$, der kleinste $72.0 \mathrm{~mm}$, durchschnittlich 74.4 mm. Also ist der totale Transversaldurchmesser bei den Formosanern etwas kleiner als bei den Europäermännern, Amerikanern und Japanermännern, und etwas grösser als bei den Neu-Pommern, Europäerweibern und Japanerweibern.
Tabelle 2. Totaler

Transversaldurchmesser.

\begin{tabular}{|c|c|}
\hline $\mathrm{mm}$ & Fälle \\
\hline $66.5-67.0$ & 2 \\
$67.1-68.0$ & 1 \\
$68.1-69.0$ & 0 \\
$69.1-70.0$ & 2 \\
$70.1-71.0$ & 2 \\
$71.1-72.0$ & 2 \\
$72.1-73.0$ & 1 \\
$73.1-74.0$ & 3 \\
$74.1-75.0$ & 2 \\
$75.1-76.0$ & 2 \\
$76.1-77.0$ & 1 \\
$77.1-78.0$ & 2 \\
$78.1-78.7$ & .2 \\
\hline
\end{tabular}

3. Vertikaldurchmesser des Arcus anterior (Tabelle 3).

Von den Vertikaldurchmessern des Arcus anterior in der Medianebene beläuft sich der grösste auf $12.0 \mathrm{~mm}$, der kleinste auf $8.9 \mathrm{~mm}$; durchschnittlich auf $10.6 \mathrm{~mm}$. Als Hauptnass ist $10.0 \mathrm{~mm}$ bis $11.0 \mathrm{~mm}$ anzunehmen. Nach

Tabelle 3.

Vertikaldurchmesser des

Arcus anterior.

\begin{tabular}{|c|c|}
\hline $\mathrm{mm}$ & Fälle \\
\hline $8.9-9.0$ & 2 \\
$9.1-10.0$ & 4 \\
$10.1-11.0$ & 6 \\
$11.1-12.0$ & 5 \\
\hline
\end{tabular}

Hasebe (13) schwankt derjenige der Europäer zwischen $9.0 \mathrm{~mm}$ und $11.0 \mathrm{~mm}$, durchschnittlich $10.1 \mathrm{~mm}$; bei den Amerikanern beträgt er durchschnittlich $10.2 \mathrm{~mm}$. Bei den Japanermänuern misst der grösste $12.0 \mathrm{~mm}$, der kleinste $9.0 \mathrm{~mm}$, durchschnittlich 10.9 mm; bei den Japanerweibern der grösste $11.0 \mathrm{~mm}$, der kleinste $8.0 \mathrm{~mm}$, durchschnittlich $9.5 \mathrm{~mm}$. Also kann man keine grosse Differenz zwischen diesen Rassen konstatieren, mit Ausnahme von japanischen Weibern, bei

denen der Durchmesser etwas kleiner als der der Formosaner ist. 
4. Vertikaldurchmesser des Arcus posterior (Tabelle 4).

Von den Vertikaldurchmessern des Arcus posterior in der Medianebene misst der grösste $13.2 \mathrm{~mm}$, der kleinste $5.8 \mathrm{~mm}$; durchschnittlich $9.9 \mathrm{~mm}$. Das Hauptmass ist $8.0 \mathrm{~mm}$ bis $11.0 \mathrm{~mm}$. Nach Hasebe (13) misst bei den Euro-

Tabelle 4.

Vertikaldurchmesser des

Arcus posterior.

\begin{tabular}{|c|c|}
\hline $\mathrm{mm}$ & Fälle \\
\hline $5.8-6.0$ & 1 \\
$6.1-7.0$ & 0 \\
$7.1-8.0$ & 3 \\
$8.1-9.0$ & 3 \\
$9.1-10.0$ & 3 \\
$10.1-11.0$ & 6 \\
$11.1-12.0$ & 3 \\
$12.1-13.0$ & 1 \\
$13.1-13.2$ & 1 \\
\hline
\end{tabular}
päern der grösste Durchmesser $13.0 \mathrm{~mm}$, der kleinste $4.0 \mathrm{~mm}$, durchschnittlich $9.9 \mathrm{~mm}$; bei den Amerikanern durchschnittlich $10.2 \mathrm{~mm}$; bei den Japanermännorn der grösste $15.0 \mathrm{~mm}$, der kleinste $9.0 \mathrm{~mm}$, durchschnittlich $10.5 \mathrm{~mm}$; bei den Japanerweibern der grösste $13.0 \mathrm{~mm}$, der kleinste $8.0 \mathrm{~mm}$, durchschnittlich $10.3 \mathrm{~mm}$. Zwischen diesen Rassen ist keine grosse Differenz bemerkbar, der von Japanern übertrifft an Grösse nur etwas den der Formosaner. Nach Hasebe (13) ist der Arcus posterior der Japaner im Verhältnis zum vorderen Bogen relativ höher als bei den Europäern. Doch ist es nicht der Fall bei den Formosanern, indem der Arcus anterior hier in den meisten Fäller etwas höher ist als der Arcus posterior.

5. Sagittaldurchmesser des Foramen vertebrale (Tabelle 5).

Von den Sagittaldurchmessern des Foramen vertebrale beläuft sich der

Tabelle 5.

Sagittaldurchmesser des

Foramen vertebrale.

\begin{tabular}{|c|c|}
\hline $\mathrm{mm}$ & Fälle \\
\hline $27.3-28.0$ & 1 \\
$28.1-29.0$ & 2 \\
$29.1-30.0$ & 4 \\
$30.1-31.0$ & 6 \\
$31.7-32.0$ & 3 \\
$32.1-33.0$ & 4 \\
$33.1-34.0$ & 0 \\
34.1 & 1 \\
\hline
\end{tabular}
grösste auf $\cdot 34.1 \mathrm{~mm}$, der kleinste auf 27.3 mm; durchschnittlich auf $30.7 \mathrm{~mm}$. Als Masse, welche am häufigsten getroffen werden, traten $29.0 \mathrm{~mm}$ bis $32.0 \mathrm{~mm}$ hervor. Nach Hasebe (13) beträgt bei den Europäern der grösste $36.0 \mathrm{~mm}$, der kleinste $28.0 \mathrm{~mm}$, durchschnittlich $32.7 \mathrm{~mm}$; bei den Amerikanern durchschnittlich $30.2 \mathrm{~mm}$; bei den Japanermännern der grösste $33.0 \mathrm{~mm}$, der kleinste 26.0 $\mathrm{mm}$, durchschnittlich $29.4 \mathrm{~mm}$; bei den Japanerweibern der grösste $31.0 \mathrm{~mm}$, der kleinste $27.0 \mathrm{~mm}$, durchschnittlich $28.2 \mathrm{~mm}$. Daher ist der betreffende Durchmesser bei den Formosanern etwas kleiner als bei den Europäern, faist gleich 
gross mit dem der Amerikaner, und etwas grösser als bei den Japanern.

6 Grösster Transversaldurchmesser des eigentlichen Foramen vertebrale (Tabelle 6).

Tabelle 6.

Grösster Transversaldurchmesser des eigentlichen Foramen vertebrale.

\begin{tabular}{|c|c|}
\hline $\mathrm{mm}$ & Fälle \\
\hline $24.1-25.0$ & 2 \\
$25.1-26.0$ & 2 \\
$26.1-27.0$ & 2 \\
$27.1-28.0$ & 5 \\
$28.1-29.0$ & 6 \\
$29.1-30.0$ & 3 \\
$30.1-31.0$ & 1 \\
$31.1-32.0$ & 0 \\
$32.1-33.0$ & 0 \\
$33.1-34.0$ & 0 \\
$34.1-35.0$ & 0 \\
$35.1-35.3$ & 1 \\
\hline
\end{tabular}

Tabelle 7. Vorderer Transversaldurchmesser des Foramen vertebrale.

\begin{tabular}{|c|c|}
\hline $\mathrm{mm}$ & Fälle \\
\hline $13.6-14.0$ & 1 \\
$14.1-15.0$ & 4 \\
$15.1-16.0$ & 4 \\
$16.1-17.0$ & 5 \\
$17.1-18.0$ & 4 \\
$18.1-19.0$ & 3 \\
$19.1-19.4$ & 1 \\
\hline
\end{tabular}

Von diesen beträgt der grösste $35.3 \mathrm{~mm}$, der kleinste $24.1 \mathrm{~mm}$; durchschnittlich $28.0 \mathrm{~mm}$. Das Hauptmass schwankt zwischen $27.0 \mathrm{~mm}$ und 29.0 mm. Nach A eby (2) misst bei den Europäermännern der grösste Durchmesser $35.5 \mathrm{~mm}$, der kleinste $29.0 \mathrm{~mm}$, durchschnittlich $31.4 \mathrm{~mm}$; bei den Europäerweibern der grösste $32.0 \mathrm{~mm}$, der kleinste $27.0 \mathrm{~mm}$, durchschnittlich $29.9 \mathrm{~mm}$. Nach Hasebe (13) beläuft sich bei den Europäern der grösste auf $32.0 \mathrm{~mm}$, der kleinste auf 27.0 mm, durchschnittlich $30.4 \mathrm{~mm}$; bei den Amerikanern durchschnittlich $28.3 \mathrm{~mm}$; bei den japanischen Männern der grösste $31.0 \mathrm{~mm}$, der kleinste $25.0 \mathrm{~mm}$, durchschnittlich $27.2 \mathrm{~mm}$; bei den japanischen Weibern der grösste $28.0 \mathrm{~mm}$, der kleinste $24.0 \mathrm{~mm}$, durchschnittlich $25.6 \mathrm{~mm}$. Also ist der betreffende Durchmesser bei den eingeborenen Formosanern etwas kleiner als bei den Europäern, fast gleich gross wie bei den Amerikanern, und etwas grösser als bei den Japanern.

7. Vorderer Transversaldurchmesser des Foramen vertebrale (Tabelle 7).

Der grösste Durchmesser misst $19.4 \mathrm{~mm}$, der kleinste $13.6 \mathrm{~mm}$; durchschnittlich $16.5 \mathrm{~mm}$. Als Hauptmass ist $15.0 \mathrm{~mm}$ bis $17.0 \mathrm{~mm}$ anzugeben. Nach Hasebe (13) beträgt bei den Europäern der grösste $20.0 \mathrm{~mm}$, der kleinste $14.0 \mathrm{~mm}$, durchschnittlich $17.7 \mathrm{~mm}$; bei den Amerikanern durchschnittlich $16.7 \mathrm{~mm}$; bei den Japanermännern der grösste $20.0 \mathrm{~mm}$, der kleinste 15.0 
mm, durchschnittlich $16.6 \mathrm{~mm}$; bei den Japanerweibern der grösste $17.0 \mathrm{~mm}$, der kleinste $13.0 \mathrm{~mm}$, durchschnittlich $14.5 \mathrm{~mm}$. Daher ist der betreffende Durchmesser bei den Formosanern etwas kleiner als bei den Europäern, etwas grösser als bei den japanischen Weibern, und fast gleich gross wie bei den japanischen Männern und Amerikanern.

Tabelle 8.

Grösste Weite der Facies articulares inferiores.

\begin{tabular}{|c|c|}
\hline $\mathrm{mm}$ & Fälle \\
\hline $40.3-41.0$ & 2 \\
$41.1-42.0$ & 4 \\
$42.1-43.0$ & 2 \\
$43.1-44.0$ & 3 \\
$44.1-45.0$ & 4 \\
$45.1-46.0$ & 5 \\
$46.1-47.0$ & 1 \\
$47.1-48.0$ & 0 \\
$48.1-49.7$ & 1 \\
\hline
\end{tabular}

Tabelle 9.

Grösste Weite der Foveae articulares superiores.

\begin{tabular}{|c|c|}
\hline $\mathrm{mm}$ & Fälle \\
\hline $42.3-43.0$ & 1 \\
$43.1-44.0$ & 0 \\
$44.1-45.0$ & 7 \\
$45.1-46.0$ & 4 \\
$46.1-47.0$ & 3 \\
$47.1-48.0$ & 1 \\
$48.1-49.0$ & 2 \\
$49.1-50.0$ & 1 \\
$50.1-51.0$ & 0 \\
$51.1-52.1$ & 1 \\
\hline
\end{tabular}

8. Grösste Weite der Facies articulares inferiores (Tabelle 8).

Von diesen Weiten misst die grösste 49.7 $\mathrm{mm}$, die kleinste $40.3 \mathrm{~mm}$; durchschnittlich 43.8 mm. Das Hauptmass beläuft sich auf $41.0 \mathrm{~mm}$ bis $46.0 \mathrm{~mm}$. Nach Hasebe (13) bewegt sich die grösste Weite bei den Europäern zwischen 44.0 $\mathrm{mm}$ und $50.0 \mathrm{~mm}$ und beträgt durchschnittlich $47.4 \mathrm{~mm}$. Bei den Amerikanern beläuft sie sich durchschnittlich auf $47.8 \mathrm{~mm}$. Bei den japanischen Männern misst, nach demselben Autor, die grösste $52.0 \mathrm{~mm}$, die kleinste $42.0 \mathrm{~mm}$, durchschnittlich $45.9 \mathrm{~mm}$; bei den japanischen Weibern die grösste $44.0 \mathrm{~mm}$, die kleinste 38.0 $\mathrm{mm}$, durchschnittlich $42.1 \mathrm{~mm}$. Diese Weite ist also bei den Formosanern ziemlich kleiner als bei den Europäern, Amerikanern und Japanermännern, und etwas grösser als bei den Japanerweibern.

9. Grösste Weite der Foveae articulares superiores (Tabelle 9).

Die grösste Weite beträgt $52.1 \mathrm{~mm}$, die kleinste 42.3, durchschnittlich $46.2 \mathrm{~mm}$. Häufigste Masse schwanken zwischen $44.0 \mathrm{~mm}$ und 47.0 mm. Im Vergleich zu der grössten Weite der Facies articulares inferiores ist die der Foveae articulares superiores in den meisten Fällen etwas grösser als dort. 
10. Sagittotransversalindex des Atlas (Tabelle 10).

Tabelle 10. Sagittotransversal- Von den oben erwähnten totalen Sagittal- und index des Atlas.

\begin{tabular}{|c|c|}
\hline Index & Fälle \\
\hline $56.1-57.0$ & 3 \\
$57.1-58.0$ & 1 \\
$58.1-59.0$ & 1 \\
$59.1-60.0$ & 6 \\
$60.1-61.0$ & 1 \\
$61.1-62.0$ & 3 \\
$62.1-63.0$ & 1 \\
$63.1-64.0$ & 1 \\
$64.1-65.0$ & 1 \\
$65.1-66.0$ & 2 \\
$66.1-66.5$ & 1 \\
\hline
\end{tabular}
Transversaldurchmessern des Atlas habe ich den betreffenden Index gerechnet. Der grösste desselben beträgt 66.5 , der kleinste 56.1 ; durchschnittlich 60.7. Der Hauptindex schwankt zwischen 59.0 und 62.0. Nach Dubreuil-Chambardel (7) beträgt er bei den europäischen Männern durchschnittlich 56.0, bei den europäischen Weibern 60.0. Nach Hasebe (13) beträgt bei den Europäern der grösste Index 61.0, der kleinste 52.4, durchschnittlich 56.9; bei den Amerikanern durchschnittlich 54.1 ; bei den Japanermännern der grösste 63.9, der kleinste 51.2, durchschnittlich 56.5 ; bei den Japanerweibern der grösste 64.8, der kleinste 52.0, durchschnittlich 58.0. Vergleicht man diese Indices miteinander, so

Tabelle 11.

Sagittotransversalindex des Foramen vertebrale.

\begin{tabular}{|l|l|}
\hline \multicolumn{1}{|c|}{ Index } & Fälle \\
\hline $80.3-81.0$ & 1 \\
$81.4-81.8$ & 4 \\
$85.6-86.0$ & 1 \\
87.1 & 1 \\
89.3 & 1 \\
90.9 & 1 \\
$91.5-91.8$ & 2 \\
92.3 & 1 \\
$93.1-93.4$ & 2 \\
$94.1-94.4$ & 2 \\
96.5 & 1 \\
$97.3-97.7$ & 2 \\
103.5 & 1 \\
112.1 & 1 \\
\hline
\end{tabular}
findet man, dass der Index der. Formosaner an Grösse denselben anderer Rassen übertrifft. Vielleicht ist dieser verhältnismässig grosse Index durch die Kleinheit des Transversaldurchmessers verursacht. Ich halte diese Tatsache für eine charakteristische Erscheinung am Formosaneratlas.

11. Sagittotransversalindex des Foramen vertebrale (Tabelle 11).

Bei den Formosanern beträgt der grösste Index 112.1, der kleinste 80.3; durchscbnittlich 91.3. Der Hauptindex schwankt zwischen 91.0 und 96.0. Den Index über 100.0 findet man in zwei Fällen. Nach Hasebe (13) beträgt bei den Europäern der grössté Index 107.1, der kleinste 77.1, durchschnittlich 93.4 ; bei den Amerikanern durchschnittlich 93.7 ; bei den Japanermännern der grösste 106.9, der kleinste 
81.8, durchschnittlich 92.5 ; bei den Japanerweibern der grösste 100.0, der kleinste 85.7, durchschnittlich 90.8. Also kann man sagen, dass der betreffende Index der Formosaner den der japanischen Weiber übertrifft und etwas kleiner ist als derjenige von Europäern, Amerikanern und japanischen Männern.

12. Anteroposteriorindex des Foramen vertebrale (Tabelle 12).

Tabelle 12.

Anteroposteriorindex des Foramen vertebrale.

\begin{tabular}{|c|c|}
\hline Index & Fälle \\
\hline 49.8 & 1 \\
51.5 & 1 \\
52.3 & 1 \\
53.8 & 1 \\
54.0 & 1 \\
55.2 & 1 \\
56.1 & 2 \\
56.7 & 1 \\
58.0 & 1 \\
59.2 & 2 \\
59.8 & 2 \\
60.2 & 1 \\
60.8 & 1 \\
62.1 & 1 \\
62.4 & 1 \\
65.6 & 1 \\
68.5 & 1 \\
69.3 & 1 \\
69.8 & 1 \\
\hline
\end{tabular}

Bei den Formosanern beträgt der grösste Index 69.8 , der kleinste 49.8 ; durchschnittlich 59.1. Der Hauptindex fiel zwischen 56.0 und 65.0. Nach Hasebe (13) beträgt bei den Europäern der grösste 69.0, der kleinste 65.0, durchschnittlich 58.5; bei den Amerikanern durchschnittlich 59.0 ; bei den japanischen Männern der grösste 72.0, der kleinste 55.6, durchschnittlich 61.4 ; bei den japanischen Weibern der grösste 64.0, der kleinste 50.0, durchschnittlich 56.7. Also kann man leicht ersehen, dass der Index bei den Formosanern fast so gross ist wie bei den Europäern und Amerikanern, etwas kleiner als bei den japanischen Männern, und ziemlich grösser als derjenige von japanischen Weibern.

B. Befunde an den einzelnen Teilen des Atlas. 1. Foramen atlantoideum.

Von dem hinteren Abschnitt der Massa lateralis entspringt manchmal eine $\mathbf{K}$ nochenbrücke nach hinten unten und ist mit der oberen Fläche des Arcus posterior kontinuierlich verwachsen, nahe seinem Wurzelteil. Dadurch entsteht hier das Foramen atlantoideum posterius, welches in sich die A. vertebralis durchziehen lässt. Auch entspringt öfters eine Knochenbrücke von dem lateralen Abschnitt der Massa nach aussen unten und ist mit der oberen Fläche des Querfortsatzes verschmolzen, wodurch dazwischen das Foramen atlantoideum laterale zu stande kommt. Doch 
sind diese Foramina nicht immer vollständig ausgebildet, sondern sehr häufig auf eine kurze Strecke unterbrochen. Besonders werden bei den Formosanern Fälle angetroffen, bei denen eine Knochenepange von dem hinteren oder lateralen Abschnitt der Massa lateralis, oder von der oberen Fläche des Arcus posterior oder des Querfortsatzes entspringt. Hasebe (13) hat unter 100 Atlanten von Japanern das vollständig geschlossene Foramen atlantoideum posterius in drei Fällen, das unvollkommene Foramen in 12 Fällen, das Foramen atlantoideum laterale in fünf Fällen und das unvollständig abgeschlossene in einem Falle gefunden. Bolk (5) konnte unter 114 Atlanten vou Europäern das Foramen atlantoideum posterius in 12 Fällen finden. Auch hat Poirier (19) 500 Atlanten von Europäern untersucht und gefunden, dass 40 Fälle beiderseits und 48 Fälle nur einerseits zur Ausbildung des Foramen atlantoideum posterius, zwei Fälle zur Ausbildung des Foramen atlantoideum laterale geneigt sind, und in zwei Fällen ein vollständiges Foramen atlantoideum posterius und laterale gleichzeitig vorhanden sind. Anutschin (3) untersuchte zwei Atlanten von Aino und beschreibt, dass sie sowohl mit dem Foramen atlantoideum posterius als auch mit dem Foramen atlantoideum laterale versehen waren.

Ich habe 22 Atlanten von Formosanern untersucht und konnte folgende Resultate gewinnen.

In einem Falle ist ein vollständiges Foramen atlantoideum laterale beiderseits ausgebildet (Fig. 1). In einem Falle ist nur an der rechten Seite ein beinahe vollkommenes, nur auf kurze Strecke unterbrochenes Foramen atlantoidoum posterius, und an der linken Seite ein mangelhaft ausgebildetes Foramen atlantoideum posterius angedeutet (Fig. 2, Fig. 3). So sind in der Majorität der Fälle die Atlanten von Formosanern stark zur Ausbildung der Foramina (Fig. 4). geneigt. Nur in acht Fällen fehlte solche Knochenspange. 
Tabelle 13.

\begin{tabular}{|c|c|c|c|c|c|c|c|c|}
\hline \multirow[t]{2}{*}{$\begin{array}{l}\text { Bezeich- } \\
\text { nung des } \\
\text { Atlas }\end{array}$} & \multicolumn{2}{|c|}{$\begin{array}{l}\text { Knochenspange ent- } \\
\text { springt vom hinteren } \\
\text { Teil der Massa } \\
\text { lateralis }\end{array}$} & \multicolumn{2}{|c|}{$\begin{array}{l}\text { Knochenspange ent- } \\
\text { springt vom lateral- } \\
\text { en Teil der Massa } \\
\text { lateralis }\end{array}$} & \multicolumn{2}{|c|}{$\begin{array}{ll}\text { Knochenspange ent- } \\
\text { springt von der } \\
\text { oberen Fläche des } \\
\text { Arcus posterior }\end{array}$} & \multicolumn{2}{|c|}{$\begin{array}{l}\text { Knochenspange ent- } \\
\text { springt von der } \\
\text { oberen Fläehe des } \\
\text { Proc. transversus }\end{array}$} \\
\hline & links & rechts & links & rechts & links & rechts & links & rechts \\
\hline 1 & + & + & - & -- & - & - & - & - \\
\hline 6 & + & - & - & + & + & + & - & - \\
\hline 7 & - & - & - & - & - & - & - & - \\
\hline 8 & - & - & - & - & - & - & - & - \\
\hline 9 & - & - & - & - & - & - & - & - \\
\hline 15 & - & - & - & - & - & + & - & - \\
\hline 19 & - & - & - & - & + & - & - & - \\
\hline 20 & - & - & - & - & - & - & - & - \\
\hline 21 & - & - & - & - & $t^{\circ}$ & - & - & - \\
\hline 25 & - & + & + & + & - & + & + & - \\
\hline 26 & + & + & + & 4 & + & + & - & - \\
\hline 28 & - & - & - & - & -- & - & - & - \\
\hline 33 & - & - & - & - & - & - & - & - \\
\hline 35 & - & - & + & - & + & - & - & - \\
\hline 36 & + & + & - & - & -- & - & - & - \\
\hline 37 & + & - & - & + & + & + & - & - \\
\hline 39 & - & - & - & - & - & - & - & - \\
\hline 40 & + & - & - & - &. & - & - & - \\
\hline 41 & - & - & + & - & + & + & - & - \\
\hline 45 & - & - & - & - & - & - & + & - \\
\hline 47 & - & - & - & - & - & - & - & - \\
\hline 50 & + & + & + & + & + & + & + & + \\
\hline Summe & 7 & 5 & 5 & 5 & 9 & 7 & 3 & 1 \\
\hline
\end{tabular}

Aus der obigen Tabelle geht hervor, dass der Knochenvorsprung am häufigsten aus der oberen Fläche des Arcus posterior, etwas seltener aus dem hinteren Teil der Massa lateralis und dem lateralen Abschnitt der Massa und am seltensten aus der oberen Fläche des Proc. transversus ausgeht. Auch kann man 
mit Recht sagen, dass bei den Formosanern das Foramen atlantoideum posterius viel stärker zur Ausbildung geneigt ist als das Foramen atlantoideum laterale.

Bei den Affen ist das Auftreten des Foramen atlantoideum posterius und laterale fast konstant, während bereits bei den Anthropoiden das letztere viel seltener vorkommt als das erstere. Phylogenetisch verschwindet, wie wohl im allgemeinen bekannt, zuerst das letztere und dann zeigt das erstere die Neigung, sich zurückzubilden. Dies ist auch bei den Formosanern der Fall. Was die Entstehung dieser Foramina anbelangt, so möchte ich sie so erklären, dass beim Foramen atlantoideum posterius die eine Knochenspange vom hinteren Teil der Massa lateralis und die andere von der oberen Fläche des Arcus posterior entspringen und beide schliesslich zu einer Brücke sich vereinigen, und dass beim Foramen atlantoideum laterale die eine Spange vom lateralen Teil der Massa und die andere von der oberen Fläche des Querfortsatzes entspringen, und zu einer Brücke zusammenfliessen, die dadurch ein Loch umgibt. Während weiterer phylogenetischer Entwicklung verschwindet beim Menschen diese Spange als Regel gänzlich, doch kann sie manchmal zurückbleiben. Die bei den Formosanern besonders häufig vorkommenden Knochenspangen sind daher als Überbleibsel des phylogenetisch verhältnismässig primitiven Gebildes zu betrachten.

\section{Processus transversus.}

Dieser Fortsatz ist bei den Formosanern mittelmässig ausgebildet. Die Spitze desselben endet in den meisten Fällen stumpf abgerundet, selten aber abgeplattet. Manchmal sind die dem Tuberculum anterius und posterius der anderen Halswirbel entsprechenden Knochenvorsprünge am Querfortsatz bemerkbar. Beide Vorsprünge treten nicht immer zugleich auf, sondern häufig fehlt der eine. Nach Poirier (19) fehlt der hintere Knochenvorsprung seltener als der vordere. An den 100 Japaneratlanten ist nach Hasebe (13) der hintere stets beiderseits vorhanden, was ich auch bei den Formosanern bestätigen konnte.

Die Form des Foramen transversum ist in den meisten Fällen elliptisch, selten kreisrund.

\section{Fovea articularis superior.}

Von deu Längen der Fovea articularis superior beträgt die grösste $25.9 \mathrm{~mm}$, die kleinste $19.8 \mathrm{~mm}$; durchschnittlich links $22.8 \mathrm{~mm}$, rechts $22.3 \mathrm{~mm}$. Die 
grösste Breite derselben, senkrecht zur Länge gemessen, beträgt $13.3 \mathrm{~mm}$, die kleinste $8.2 \mathrm{~mm}$; durchschnittlich links $10.9 \mathrm{~mm}$, rechts $10.3 \mathrm{~mm}$. Frizzi (11) betont, dass bei den Neu-Pommern das Mass der Fovea an der rechten Seite etwas grösser ist als an der linken. Bei den Formosanern ist jedoch die linke ein weinig grösser als die rechte. Der grössere Durchmesser ist schräg gestellt, so dass die beiderseitigen Längsachsen einige Zentimeter vor dem Tuberculum anterius einander schneiden.

Die Fovea wird manchmal durch eine quere Spalte in zwei Felder geteilt. Krause (17) ist der Meinung, dass diese Spaltung selten auftritt. Dagegen betrachtet Disse (6) dies als eine öfters vorkommende Erscheinung. Poirier (19) beobachtet dieselbe etwa in einem Zehntel der untersuchten Fälle. Hasebe (13) fand dies an sieben Europäeratlanten in vier Fällen, während er es an 100 Japanern niemals nachweisen konnte. Ich konnte an 22 Formosaneratlanten in zwei Fällen eine vollständige Trennung der Fovea finden. Doch ist die unvollständige Trennung äusserst häufig zu sehen, welche teils beiderseitig, teils nur an einer Seite auftritt. Somit kann man sagen, dass bei den Formosanern die Fovea articularis superior vielmehr geneigt ist, sich in zwei Felder zu trennen.

\section{Facies articularis inferior.}

Gegenüber vorhergehender Fovea ist die Facies articularis inferior wenig ausgehöhlt, selten dagegen etwas vorgewölbt. Sie ist am häufigsten abgerundet dreieckig, sehr oft fast kreisrund und selten elliptisch. Ihre grösste Länge beträgt $21.0 \mathrm{~mm}$, die kleinste $15.0 \mathrm{~mm}$; durchschnittlich links $17.8 \mathrm{~mm}$, rechts 17.9 mm. Die grösste Breite beträgt $17.3 \mathrm{~mm}$, die kleinste $13.5 \mathrm{~mm}$; durchschnittlich beiderseits $15.5 \mathrm{~mm}$.

\section{Tuberculum anterius et posterius.}

Das Tuberculum anterius ist fast in der Hälfte der Fälle sehr schwach angedeutet. In den gut ausgebildeten Fällen liegt es immer nahe dem unteren Rand des Arcus anterior.

Das Tuberculum posterius ist ziemlich mannigfaltig ausgeprägt. Unter 21 Atlanten fehlt es in sechs Fällen gänzlich, in sieben Fällen ist eine Crista entlang der Höhe des Arcus und in drei Fällen ein etwas tiefer Einschnitt anstatt des 
Tuberculum auggebildet. Das Tuberculum posterius im genaueren Sinne trat bei mir nur in sechs Fällen auf. Falls das Tuberculum entsteht, ist es gegenüber dem Tuberculum anterius am oberen Rande des Arcus posterior gelegen.

6. Fovea dentis.

Die Fovea dentis ist bei den Formosanern teils rundlich, teils elliptisch dargestellt. Die Grösse desselben ist schwankend. In den meisten Fälleц nimmt die Fovea ihre Lage entlang der ganzen Höhe des Arcus anterior ein. Selten ist sie mehr dem unteren Rand des Arcus genähert gelegen. Ihre grösste Länge beträgt durchschnittlich $8.3 \mathrm{~mm}$, die Breite durchschnittlich $9 \cdot 2 \mathrm{~mm}$.

7. Höcker für das Lig. trangversarium atlantis.

An der inneren Fläche der Massa lateralis ist immer ein Höcker nebst einer Grube vorhanden für Ursprung oder Ansatz des Lig. transversarium atlantis, das den Zahn des Epistropheus von hinten umfasst. Sein längerer Durchmesser steht von hinten aussen oben nach vorn innen unten gerichtet. In $45.2 \%$ der untersuchten Fälle berührt das untere Ende dieses Höckers die Facies articularis inferior, in $4.8 \%$ berührt sein oberes Ende die Fovea articularis superior und in $16.7 \%$ berühren seine beiden Enden je die Fovea articularis superior und inferior, während er in $32.9 \%$ niemals diese Gelenkflächen berührt. Der lange Durchmesser des Höckers beträgt durchschnittlich links $7.6 \mathrm{~mm}$, rechts $7.9 \mathrm{~mm}$, die Breite durchschnittlsch beiderseits $4.7 \mathrm{~mm}$ Die Tiefe der Grube nahe diesem Höcker ist bald beträchtlich, bald aber sehr gering ausgebildet.

\section{Arcus posterior.}

Dass an der unteren und oberen Fläche des Arcus posterior, nahe der Medianlinie, eine sehr lang gestreckte spindelförmige Gelenkfläche vorhanden ist, wurde schon von Weigner (24) beschrieben. Auch konnte ich dies bei den Formosanern mit Sicherheit nachweisen. Die Gelenkfläche an der unteren Arcusfläche artiknliert nit der oberen Fläche des Arcus des Epistropheus. Bei den Formosanern kommt sie fast in der Hälfle der Fälle, bald beiderseits, bald nur einerseits, vor. Die Gelenkfläche an der oberen Arcusfläche artikuliert mit dem Hinterlauptbein. Die letztere kommt viel weniger als die erstere vor, nur in einem Viertel der untersuchten Fälle. Ihre Form ist bald spindelförmig, bald elliptisch oder linear. 
9. Processus odontoideus atlantis.

Ich konute, obgleich nur in einem Falle, ein Paar hornartiger Knochenvorsprünge oberhalb des Tuberculum anterius konstatieren. Halbertsma hat den Fortsatz Proc. opercularis atlantis genannt. Funke (12) bezeichnet ihn als Proc. odontoideus atlantis. Poirier (18) schreibt: "Cette apophyse s'étendait du bord supérieur de l'arc antérieur de l'atlas, montait en avant de lapophyse odontoide et se recurbait en crochet pour sarticuler par sa face inférieur avec le sommet de l'apophyse odontoide. Sur un grand nombre d'atlas nous avons constaté une cerie de rugosités irrégulaires; surmontant le bord supérieur de la fossette articulaire odontoidienne, et provenant, il n'a pas doute, de l'ossification de ligament occipitoodontoidien." Es ist sehr fraglich, ob es sich um eine aus der Bildungsgeschichte des Atlas und Epistropheus herstammende Anomalie oder um etwas Pathologisches handelt. Über seine Entstehung schliesst sich Tschugunow (23) mehr oder weniger an die von Poirier (19) gegebene Erklärung an, nach welchem der Fortsatz in denjenigen Fällen entsteht, bei denen der Bandapparat hier durch irgend eine pathologische Veränderung, z. B. Spondylitis deformans, verknöchert. Er soll nach Tschugunow (23) nicht selten bei alten Individuen beider Geschlechter angetroffen werden.

\section{Assimilation des Atlas.}

Die Verschmelzung der Halswirbel kommt sehr oft vor. Seitdem 1767 Morgagni die Assimilation des Atlas, die durch Gelenkankylose verursacht wurde, angegeben hat, haben Sandifort (1783), Leveling (1787), Gurt (1853) u.a. auch darauf ihre Aufmerksamkeit gelenkt. Hinsichtlich deren Entstehung ist aber bis heute keine entscheidende Meinung vorhanden. Danach studierten auch Bockshammer (1862), R. Virchow (1876), Sommer (1883), Adachi (1889), Langerhans (1890), Macalister (1893), Regnault (1900), Dorello (1900), Szawlowsky (1901, 1902), Swjetschńikow (1906), Bolk (1906), Kubo (1906), Kollmann (1905, 1907); Hasebe (1913) u.a. entweder entwicklungsgeschichtlich, pathologisch-anatomisch, oder anthropologisch die Sache ziemlich eingehend. Unter diesen Autoren hat Swjetschnikow die Entstehung der Assimilation in drei Gruppen eingeordnet: 1.'Acquirierte Fälle durch Tuberkulose, Syphilis, Arthritis deformans, Arthritis adhaesiva chronica usw, 2. in Utero acquirierte Fälle durch Druck bei abnormem Becken, und 3. rein 
kongenitale Fälle, wobẹi der Verwachsungsprozess der in den Schädel eingegangenen Sklerotome abnormerweise auch das Sklerotom des ersten Halssegmentes, aus dem der Atlas hervorgeht, teilweise mitergriffen hat, ein Prozess, der in der Frühperiode der embryonalen Entwicklung sich abspielt.

Der Grad der Assimilation ist ziemlich mannigfaltig; bald beginnt sie im Atlantooccipitalgelenk selbst und bringt keine Formveränderang des Atlas mit sich, bald aber assimiliert der Atlas sehr hochgradig und sein Arcus posterior fehlt fast gänzlich. Je stärker der Atlas mit dem Hinterhanptbein assimiliert, desto hochgradiger ist im allgemeinen seine Reduktion.

Die folgende Tabelle gibt die Häufigkeit der Assimilation an :

Tabelle 14.

\begin{tabular}{l|c|c|l|l}
\hline Rasse & Untersuchte Zahl & Fälle & $\%$ & Autor \\
\hline Europäer & 700 & - & 1.5 & Morselli \\
Europäer & 436 Schädel & 2 & 0.45 & Swjetschnikow \\
Europäer & 1055 Schädel & - & 0.4 & Russel \\
Europäer & 20 S0 Schäde & 4 & 0.2 & Friedlowsky \\
Europäer & $\ldots \ldots . .$. & - & 0.14 & Macalister \\
Europäer & $\ldots . . . .$. & - & 0.84 & Lombroso \\
Europäer & $\ldots \ldots . .$. & - & 2.0 & Sommer \\
Japaner & 145 Schädel & 4 & 2.8 & Adachi \\
Japaner & 100 Wirbelsäule & 1 & 1.0 & Kubo \\
Japaner & 181 Wirbelsäule & 2 & 1.1 & Hasebe \\
Japaner & 400 Schädel & 2 & 0.5 & Hasebe \\
Eingeborene & 45 Schädel & 1 & 2.2 & Tsueaki \\
Formosaner & & & & \\
\hline
\end{tabular}

Im allgemeinen tritt die Assimilation des Atlas bei den Japanern ziemlich häufiger anf als bei den Europäern. Um deren Häufigkeit bei den Formosanern sicher zu bestimmen, bedarf es noch weiterer Untersuchungen an vielen Materialien. Doch möchte ich aus der obigen Tabelle vermuten, dass die Assimilation hier ebenso hänfig wie bei den Japanern vorkommt.

Bei meinem Falle ist die Fovea articularis superior mit dem Condylus occipitalis fest.verwachsen. Rückbildung des Atlas ist nicht bemerkbar, indem seine Form nnd Gröse normal und symmetrisch sich verhalten. Der Areus anterius und posterius und die Massa lateralis bleiben ganz isoliert zurück. 
Sowohl das Spatium atlantooccipitale anterius als auch posterius sind in der ganzen. Ausdehnung ziemlich weit erhalten, doch etwas enger als die Norm (Fig. 5).

C. Gewicht des Atlas.

Wenn auch die Wägung des Knochens in der Anthropologie bis heute noch nicht allgemein gebränchlich ist, so ist doch schon genug bewiesen, dass sie die Resultate der gewöhnlichen Messungen wohl ergänzen kann. Aus diesem Grund wägt Zoja (27) das Gewicht des Atlas von Pavianen und gibt an, dass das Gewicht. bei Männchen durchschnittlich $9.6 \mathrm{~g}$, bei Weibchen durchschnittlich $6.9 \mathrm{~g}$ beträgt. Auch hat Zanolli (26) den Atlas von 18 Männern und 24 Weibern gewogen. Dabei schwankte das Gewicht bei den Männern zwischen 9.0 und $12.0 \mathrm{~g}$, bei den Weibern zwischen 5.0 und 11.0 g. Doch beeinflussen Art und Weise der Mazeration, wie Grad der Entfettung das Knochengewicht in erheblichem Masse. Mein Material stammt meistens aus in üblicher Weise mazerierten Skeleten.

Tabelle 15.

Gewicht des Atlas.

\begin{tabular}{|c|c|}
\hline gr. & Fälle \\
\hline $10.2-11.0$ & 2 \\
$11.1-12.0$ & 4 \\
$12.1-13.0$ & 5 \\
$13.1-14.0$ & 5 \\
$14.1-15.0$ & 3 \\
15.6 & 1 \\
\hline
\end{tabular}

Das Gewicht des Atlas bei den Formosanern verhält sich wie folgende Tabelle :

Aus dieser Tabelle geht hervor, dass das grösste Gewicht des Atlas $15.6 \mathrm{~g}$, das kleinote $10.2 \mathrm{~g}$ beträgt. Am häufigsten ist das Gewicht von $11.0 \mathrm{~g}$ bis $14.0 \mathrm{~g}$, durchschnittlich $12.9 \mathrm{~g}$. Die Schwankungsbreite ist daher bei den Formosanern ziemlich klein.

D. Asymmetrie des Atlas.

Der Atlas bei den Formosanern ist wie die anderen Knochenteile nicht immer symmetrisch ausgebildet, sondern stellt manchmal ziemlich merkliche Asymmetrie dar. Unter 21 Atlanten waren nur vier Atlanten ganz symmetrisch, während sieben Atlenten in verschiedenem Grad mach links abweichen, zehn dagegen stark nach rechts ablenken (Fig. 6).

\section{Epistropheus.}

\section{A. Ergebniese der Messungen.}

1. Vordere ganze Höhe (Tabelle 16).

Von den vorderen ganzen Höhen des zweiten Halswirbels der Formosaner beträgt die gröseto $40.2 \mathrm{~mm}$, die kleinote $33.4 \mathrm{~mm}$; durchschnittlich $36.7 \mathrm{~mm}$. 
Tabelle 16.

Vordere ganze Höhe.

\begin{tabular}{|c|c|}
\hline $\mathrm{mm}$ & Fälle \\
\hline $33.4-34.0$ & 3 \\
$34.1-35.0$ & 1 \\
$35.1-36.0$ & 3 \\
$36.1-37.0$ & 3 \\
$37.1-38.0$ & 2 \\
$38.1-39.0$ & 4 \\
$39.1-40.2$ & 3 \\
\hline
\end{tabular}

Tabelle 17.

Hintere ganze Höhe.

\begin{tabular}{|c|c|}
\hline $\mathrm{mm}$ & Fälle \\
\hline $29.0-30.0$ & 3 \\
$30.1-31.0$ & 2 \\
$31.1-32.0$ & 4 \\
$32.1-33.0$ & 2 \\
$33.1-34.0$ & 0 \\
$34.1-35.0$ & 5 \\
$35.1-36.7$ & 2 \\
\hline
\end{tabular}

Tabelle 18.

Vordere Höhe des Dens.

\begin{tabular}{|c|c|}
\hline $\mathrm{mm}$ & Fälle \\
\hline $18.4-19.0$ & 3 \\
$19.1-20.0$ & 4 \\
20.121 .0 & 4 \\
$21.1-22.0$ & 8 \\
22.2 & 1 \\
\hline
\end{tabular}

Das Hauptmass schwankt zwischen $35.0 \mathrm{~mm}$ und $39.0 \mathrm{~mm}$. Berechnet man vorläufig deren Mittelzahl aus Frizzis Tabelle, so beträgt sie bei den Neu-Pommern durchschnittlich $34.0 \mathrm{~mm}$. Nach Hasebe (13) beträgt sie durchschnittlich bei den Japanermännern $37.1 \mathrm{~mm}$, bei den Japanerweibern $33.8 \mathrm{~mm}$; bei den Europäern im Durchschnitt $39.3 \mathrm{~mm}$. Also steht sie bei Formosanern gerade zwischen der von Japanermännern und Europäern einerseits und der von Japanerweibern und Neu-Pommern andererseits.

\section{Hintere ganze Höhe (Tabelle 17).}

Die hintere ganze Höhe zeigt eine Schwankungsbreite von $29.0 \mathrm{~mm}$ bis $36.7 \mathrm{~mm}$. Durchschnittlich beträgt sie $32.7 \mathrm{~mm}$. Als Hauptmasse treten $31.0 \mathrm{~mm}$ bic $35.0 \mathrm{~mm}$ hervor. Bei den Japauermännern beträgt sie nach Hasebe durchschnittlich $32.8 \mathrm{~mm}$, bei den Japanerweibern 29.8 mm. Also ist die der Formosaner fast gleich gross wie bei Japanermännern, aber etwas grösser als die der Japanerweiber.

3. Vordere Höhe des Dens (Tabelle 18).

Die vordere Höhe des Dens beläuft sich auf $18.4 \mathrm{~mm}$ bis $22.2 \mathrm{~mm}$, dnrchschnittlich $20.5 \mathrm{~mm}$. Die Masse, welche am häufigaten gewonnen wurden, sind $19.0 \mathrm{~mm}$ bis $22.0 \mathrm{~mm}$. Nach Hasebe (13) beträgt dieselbe durchschnittlich bei den Japanermännern $17.9 \mathrm{~mm}$, bei den Japanerweibern $16.5 \mathrm{~mm}$. Demnach ist die Zahl bei den Formosaneru viel grösser als bei den Japanern. 
4. Vordere Höhe des Körpers (Tabelle 19).

Tabelle 19.

Die vordere Höhe des Körpers hat eine Vordere Höhe des Körpers. Schwankungsbreite zwischen $13.9 \mathrm{~mm}$ und 19.1

\begin{tabular}{|c|c|}
\hline $\mathrm{mm}$ & Fälle \\
\hline $13.9-15.0$ & 3 \\
15.116 .0 & 5 \\
$16.1-17.0$ & 5 \\
$17.1-18.0$ & 3 \\
$18.1-19.1$ & 3 \\
\hline
\end{tabular}
$\mathrm{mm}$, durchschnittlich beträgt sie $16.4 \mathrm{~mm}$. Als Hauptmasse bestehen $15.0 \mathrm{~mm}$ bis $18.0 \mathrm{~mm}$. Nach Hasebe (13) beträgt sie bei den Japanermännern durchschnittlich $19.3 \mathrm{~mm}$, bei den Japanerweibern $17.3 \mathrm{~mm}$. Also ist die der Formosaner ziemlich kleiner als die der Japaner.

Solche Höhendifferenz des Dens und des Körpers zwischen den Japanern und Formosanern wird verursacht durch die niedrige Stellung eines Höckerchens, welches in der mittleren Höhe der vorderen Medianlinie des zweiten Halswirbels sich befindet. Demnach ist bei den Formosanern die vordere Denshöhe grösser und die vordere Körperhöhe kleiner als bei Japanern. Ich habe, nach Hasebe, die Grenze zwischen dem Körper und dem Dens direkt auf die Spitze des betreffenden Höckerchens gelegt. Von diesem Höckerchen ans zieht immer eine ziemlich

Tabelle 20.

Ganze Breite. .

\begin{tabular}{|c|c|}
\hline $\mathrm{mm}$ & Fälle \\
\hline $46.7-47.0$ & 1 \\
$47.1-48.0$ & 0 \\
$48.1-49.0$ & 1 \\
$49.1-50.0$ & 0 \\
$50.1-51.0$ & 1 \\
$51.1-52.0$ & 3 \\
$52.1-53.0$ & 3 \\
$53.1-54.0$ & 2 \\
$54.1-55.0$ & 2 \\
$55.1-56.0$ & 1 \\
$56.1-57.0$ & 1 \\
$57.1-57.8$ & 3 \\
\hline
\end{tabular}

deutlich ausgeprägte Leiste beiderseits horizontal nach aussen hin und kreuzt sich rechtwinklig mit der medianen Leiste. Also liegt das Höckerchen in der Kreuzungastelle beider Leisten. Die horizontale Leiste ist nichts anderes als die Verwachsungsstelle des Körpers mit dem Zahn. An der dem Höckerchen entsprechenden Stelle der dem Wirbelloch zugekehrten Fläche liegt bei den Formosanern stets ein deutlich ausgehöhltes Grübchen, woraus eine ziemlich starke Leiste nach unten bis zum unteren Körperrande läuft.

5. Ganze Breite (Tabelle 20).

Bei den 20 zweiten Halswirbeln schwankte die ganze Breite zwischen $46.7 \mathrm{~mm}$ und $57.8 \mathrm{~mm}$; durchschnittlich betrug sie $53.3 \mathrm{~mm}$. Am meisten wurde gemessen $51.0 \mathrm{~mm}$ bis $55.0 \mathrm{~mm}$. Bei den 
Europäern beträgt die ganze Breite nach $\mathrm{D}_{\text {wight }}(8,9,10)$ durchschnittlich 58.0 bei $55.0 \mathrm{~mm}$. Nach Hasebe (13) beträgt sie bei den Japanermännern durchschnittlich $57.0 \mathrm{~mm}$, bei den Japanerweibern $50.4 \mathrm{~mm}$, und bei den Europäern $56.1 \mathrm{~mm}$. Berechnet man vorläufig die Mittelzahl aus der Frizzischen Tabelle, so beläuft sie sich bei den Neu-Pommern durchschnittlich auf $48.3 \mathrm{~mm}$. Also ist die ganze Breite der Formosaner etwas kleiner als die der Japanermänner und Europäer, aber etwas grösser als die der Japanerweiber und Neu-Pomner.

Tabelle 21. Grösster Sagittaldurchmesser des Dens.

\begin{tabular}{|c|c|}
\hline $\mathrm{mm}$ & Fälle \\
\hline $10.2-11.0$ & 8 \\
$11.1-12.0$ & 7 \\
$12.1-12.4$ & 5 \\
\hline
\end{tabular}

Tabelle 22. Transversaldurchmesser des Dens.

\begin{tabular}{|c|r|}
\hline $\mathrm{mm}$ & Fälle \\
\hline $9.2-10.0$ & 5 \\
$10.1-11.0$ & 12 \\
$11.1-12.0$ & 2 \\
13.5 & 1 \\
\hline
\end{tabular}

Tabelle 23. Distanz der äussersten Ausladung der Proc. articulares superiores.

\begin{tabular}{|c|c|}
\hline $\mathrm{mm}$ & Fälle \\
\hline $40.7-41.0$ & 1 \\
$41.1-42.0$ & 1 \\
$42.1-43.0$ & 6 \\
$43.1-44.0$ & 1 \\
$44.1-45.0$ & 2 \\
$45.1-46.0$ & 6 \\
$46.1-46.7$ & 2 \\
\hline
\end{tabular}

6. Grösster Sagittaldurchmesser des Dens (Tabelle 21).

Was den grössten Sagittaldurchmesser des. Dens anlangt, so erhalten wir eine Zahl, die sich zwischen $10.2 \mathrm{~mm}$ und $12.4 \mathrm{~mm}$ bewegt, und durchschnittlich $11.3 \mathrm{~mm}$ beträgt. Häufigstes Mass war 10.0 bis $11.0 \mathrm{~mm}$. Bei den Japanermännern beträgt er nach Hasebe (13) durchschnittlich $10.7 \mathrm{~mm}$, bei den Japanerweibern 10.5 mm. Damit ist er bei den Formosanern etwas grösser als bei den Japanern.

7. Transversaldurchmesser des Dens (Tabelle 22).

Der Transversaldurchmesser des Dens. schwankt zwischen $9.2 \mathrm{~mm}$ und $13.5 \mathrm{~mm}$; durchschnittlich $10.6 \mathrm{~mm}$. Am meisten trifft man das Mass 10.0 mm. Nach Hase be (13) beträgt er bei den Japanern durchschnittlich $19.5 \mathrm{~mm}$, bei den Japanerweibern $9.8 \mathrm{~mm}$; so ist er bei den Formosanern fast gleich gross wie bei Japanermännern, aber etwas grösser als bei den Japanerweibern.

8. Distanz zwischen den äussersten Ausladungen der Processus articulares superiores

(Tabelle 23).

Das Maximum der Distanz der äussersten Ausladung der Processus articulares superiores 
beträgt $46.7 \mathrm{~mm}$, das Minimum $40.7 \mathrm{~mm}$, durchschnittlich $44.1 \mathrm{~mm}$. Als Hauptmass dürfte $42.0 \mathrm{~mm}$ bis $\mathbf{4 6 . 0} \mathrm{mm}$ zu nennen sein. Nach Ha sebes Tabelle beträgt sie bei den Japanermännern durchschnittlich $46.2 \mathrm{~mm}$, bei den Japanerweibern $42.6 \mathrm{~mm}$, und bei den Europäern $47.1 \mathrm{~mm}$. So ist die der Formosaner etwas grôsser als die der Japanerweiber, aber etwas kleiner als die der Japanermänner und Europäer.

9. Distanz zwiechen den äussersten Ausladungen der Processus articulares

Tabelle 24. Distanz der äussersten Ausladung der Proc. articulares inferiores.

\begin{tabular}{|c|c|}
\hline $\mathrm{mm}$ & Fälle \\
\hline $42.8-43.0$ & 1 \\
$43.1-44.0$ & 3 \\
$44.1-45.0$ & 6 \\
$45.1-46.0$ & 2 \\
$46.1-47.0$ & 2 \\
$47.1-48.0$ & 0 \\
$48.1-49.0$ & 2 \\
\hline
\end{tabular}
inferiores (Tabelle 24).

Die grösite Distanz beträgt bei den Formosanern $49.0 \mathrm{~mm}$, die kleinste $42.8 \mathrm{~mm}$, durchschnittlich $45.1 \mathrm{~mm}$. Als Hauptmasse sind 44.0 $\mathrm{mm}$ bis $46.0 \mathrm{~mm} \mathrm{zu}$ nennen. Die betreffende Distanz beträgt, nach Hase be (13), bei den Japanermännern durchschnittlich $46.9 \mathrm{~mm}$, bei den Japanerweibern $42.9 \mathrm{~mm}$ und bei den Europäern $49.7 \mathrm{~mm}$. Also steht die von Formosanern gerade zwischen den von Japanerwëibern und Japanermännern, und ist ziemlich kleiner als die der Europäer.

10. Sagittaldurchmesser der unteren Endfläche (Tabelle 25).

Tabelle 25.

Sagittaldurchmesser der unteren Endfläche.

\begin{tabular}{|l|c|}
\hline \multicolumn{1}{|c|}{$\mathrm{mm}$} & Fälle \\
\hline $15.2-16.0$ & 4 \\
$16.1-17.0$ & 8 \\
$17.1-18.0$ & 6 \\
18.4 & 1 \\
\hline
\end{tabular}

Der Sagittaldurchmesser der unteren Endfläche zeigt bei den Formosanern eine Schwankungsbreite von $15.2 \mathrm{~mm}$ bis $18.4 \mathrm{~mm}$, durchschnittlich $16.7 \mathrm{~mm}$. Das Hauptmass schwankt zwischen $16.0 \mathrm{~mm}$ und $18.0 \mathrm{~mm}$. Bei den Neu-Pommern beträgt er nach Frizzi (11) durchschnittlich $14.4 \mathrm{~mm}$. Nach Hasebe (13) beträgt er bei den Japanermännern dnrchschnittlich $15.7 \mathrm{~nm}$, bei den Japanerweibern $14.6 \mathrm{~mm}$.

Alro ist die Zahl bei den Formosanern etwas grösser als bei den Japanern und den Nen-Pommern. 
11. Transverealdurchmesser der unteren Endfläche (Tabelle 26).

Dieser beläuft sich bei den Formosanern auf $17.5 \mathrm{~mm}$ bis $21.3 \mathrm{~mm}$, durch-

Tabelle 26.

Transversaldurchmesser der unteren Endfläche.

\begin{tabular}{|c|c|}
\hline $\mathrm{mm}$ & Fälle \\
\hline $17.5-18,0$ & 4 \\
$18.1-19.0$ & 8 \\
$19.1-20.0$ & 4 \\
$20.1-21.3$ & 3 \\
\hline
\end{tabular}

Tabelle 27.

Sagittaldurchmesser des Foramen vertebrale.

\begin{tabular}{|l|c|}
\hline \multicolumn{1}{|c|}{$\mathrm{mm}$} & Fälle \\
\hline 16.0 & 1 \\
$18.0-19.0$ & 6 \\
$19.1-20.0$ & 4 \\
$20.1-21.0$ & 1 \\
$21.1-22.0$ & 3 \\
$22.1-23.2$ & 2 \\
\hline
\end{tabular}

Tabelle 28.

Transversaldurchmesser des Foramen vertebrale.

\begin{tabular}{|c|c|}
\hline $\mathrm{mm}$ & Fälle \\
\hline $20.6-21.0$ & 3 \\
$21.1-22.0$ & 3 \\
$22.1-23.0$ & 3 \\
$23.1-24.0$ & 4 \\
$24.1-25.0$ & 3 \\
$25.1-25.6$ & 2 \\
\hline
\end{tabular}

schnittlich $18.8 \mathrm{~mm}$. Das Hauptmass fiel zwischen $18.0 \mathrm{~mm}$ und $20.0 \mathrm{~mm}$. Nach Frizzi (11) beträgt er bei den Neu-Pommern durchschnittlich 16.4 $\mathrm{mm}$. Bei den Japanermännern beträgt er nach Hasebe durchschnittlich $19.7 \mathrm{~mm}$, bei den Weibern $18.7 \mathrm{~mm}$. So ist er bei Formosanern etwas kleiner als bei Japanermännern, und fast gleich gross wie bei Japanerweibern.

Die Form der unteren Endfläche des zweiten Halswirbels ist daher bei den Formosaneru mehr zur Bildung der rundlichen Form geneigt als bei den Japanern und Neu-Pommern.

12. Sagittaldurchmesser des Foramen vertebrale (Tabelle 27).

Derselbe bewegt sich bei den Formosanern zwischen $16.0 \mathrm{~mm}$ und $23.2 \mathrm{~nm}$, durchschnittlich beträgt er $19.8 \mathrm{~mm}$. Das Hauptmass steht zwischen $18.0 \mathrm{~mm}$ und $22.0 \mathrm{~mm}$. Nach Hasebe (13) beträgt er bei den Japanermännern durchschnittlich $15.7 \mathrm{~mm}$, bei den Japanerweibern 15.4 mm. Demnach kann man sagen, dass dieser Durchmesser bei den Formosanern viel grösser ist als bei den Japanern.

13. Transversaldurchmesser des Foramen vertebrale (Tabelle 28 ).

Der grösste Durchmesser bei den Formosanern beträgt $25.6 \mathrm{~mm}$, der kleinste $20.6 \mathrm{~mm}$; durchschnittlich $22.9 \mathrm{~mm}$. Das Hauptmass schwankt zwischen $21.0 \mathrm{~mm}$ und 25.0 mm.--Bei den Japanermänneru beträgt er, nach Hasebe (13), durchschnittlich $21.9 \mathrm{~mm}$, bei den Weibern 21.6 
mm. Daher ist der betreffende Durchmesser bei den Formosanern etwas grösser als bei den Japanern. Kurz, man kann mit Recht sagen, dass das Foramen vertebrale bei den Formosanern viel gröeser ausgebildet ist als bei den Japanern.

B. Befunde an einigen Teilen des Epistropheus.

1. Facies articularis anterior.

Die Facies articularis anterior ist bei den Formosanern immer sehr breit ausgebildet. Unter 20 Epistrophei ist in 13 Fällen die vordere Gelenkfläche elliptisch, in 7 Fällen fast kreisrund ausgebildet. Bei 20 Epistrophei schwankte die Länge zwischen $9.4 \mathrm{~mm}$ und $12.5 \mathrm{~mm}$, durchschnittlich beträgt sie $10.9 \mathrm{~mm}$. Als Maximum ihrer Breite fand ich $10.0 \mathrm{~mm}$, als Minimum $7.1 \mathrm{~mm}$, durchschnittlich $8.5 \mathrm{~mm}$.

2. Facies articularis superior.

Die Facies articularis superior ist gewöhnlich bei den Formosanern rundlich dreieckig, selten fast kreisrund, noch seltener nierenförmig gestaltet, und zwar war sie unter 40 Facies der 20 Epistrophei in 30 Fällen rundlich dreieckig, in 6 Fällen beinahe kreisrund und in 5 Fällen nierenförmig. Hasebe (13) hat eine kreisrunde obere Gelenkfäche nur einmal unter 30 Japanerepistrophei gefunden. Sie zeigt in der Frontalrichtung eine sehr leichte Konkavität und in der Sagittalrichtung eine schwache Konvexität. Die beiderseitigen Facies konvergieren medianwärts nach oben. Der Konvergenzwinkel bewegt sich zwischen $124^{\circ}$ und $141^{\circ}$, durchschnittlich $134^{\circ} .7$. Nach Hasebe (13) bewegt sich der Winkel bei den Japanern gewöhnlich um 140 . Nach Frizzi (11) schwankt er bei den Neu-Pommern zwischen $135^{\circ}$ und $145^{\circ}$. Also ist die obere Gelenkfläche bei den Formosanern etwas steiler geneigt als bei den Japanern.

Die Länge der Facies articularis superior sohwankt bei den Formosanern zwischen $16.4 \mathrm{~mm}$ und $20.4 \mathrm{~mm}$, durchschnittlich links $18.5 \mathrm{~mm}$, rechts 18.5 $\mathrm{mm}$; die Breite $14.8 \mathrm{~mm}$ bis $17.9 \mathrm{~mm}$, durchschnittlich links $16.6 \mathrm{~mm}$, rechts $16.3 \mathrm{~mm}$.

\section{Facies articularis inferior.}

Die Facies articularis inferior ist für gewöhnlich rundlich dreieckig, selten elliptisch, noch seltener fast kreisrund gestaltet. Sie zeigt sowohl in der Frontalals aush in der Sagittalrichtung eine schwache Konkavität. So ist sie in $55.1 \%$ 
der untersuchten Fälle rundlich dreieckig, in $27.6 \%$ elliptisch und in $17.2 \%$ rundlich ausgebildet. Die beiderseitigen Facies konvergieren medianwärts nach unten vorn. Der Konvergenzwinkel bewegt sich zwischen $167^{\circ}$ und $137^{\circ}$, durchschnittlich beträgt er $155^{\circ} .8$. Die Länge der unteren Gelenkfläche misst bei den Formosanern $9.6 \mathrm{~mm}$ bis $15.0 \mathrm{~mm}$, durchschnittlich links $12.6 \mathrm{~mm}$, rechts $12.1 \mathrm{~mm}$. Von den Breiten beträgt die grösste $12.9 \mathrm{~mm}$, die kleinste 7.8 $\mathrm{mm}$, durchschnittlich links $10.0 \mathrm{~mm}$, rechts $9.9 \mathrm{~mm}$.

\section{Processus spinosus.}

In bezug auf den Processus spinosus hat Frizzi (11) unter sieben NeuPommernepistrophei nur in einem Falle einen gabelig zweigeteilten Dornfortsatz konstatiert. Bei den Formosanern konnte ich unter 17 Epistrophei nur in drei Fällen den Dornfortsatz vollständig gespalten finden. In den übrigen Fällen waren die beiden Zacken durch Knochenmasse verbunden und bildeten einen annähernd geraden Rand. Auf dem Dornfortsatz ist ein ziemlich mächtiger Knochenkamm vorhanden, dessen Kante gegen das Foramen vertebrale zu knopfartig verdiclit ist. 


\section{Benutzte Literatur.}

1. Adachi, B., Katalog der Präparate in der Sammlung des anatomischen Institutes, medizinische Abteilung der dritten Hochschule. OkayamaIgakkwai-Zasshi, Heft 117, 118 und 119. 1889. (Japanisch)

2. Aeby, Ch., Die Altersverschiedenheiten der menschlichen Wirbelsäule. Arch. f. Anat. u. Entw. 1879.

3. Anutschin, D.N., Der Stamm der Ainos. 1876.

4. Bolk, L., Kurze Mitteilungen aus dem anatomischen Irstitut zu Amsterdam. II. Über eine Wirbelsäule mit nur sechs Halswirbeln. Morph. Jahrb. Bd.29. 1900 .

5. Bolk, L., Zur Frage der Assimilation des Atlas am Schädel beim Menschen. Anat. Anz. Bd.28. 1906.

6. Disșe, J., Die Wirbelsäule. Bardelebens Handbuch der Anatomie. Skeletlehre. Abt. 1. Jena. 1896.

7. Dubreuil-Chambardel, Variations sexuelles de l'atlas. Bull. Soc. Anthropol. Paris. Série 5. T.8. 1907.

8. Dwight, Th., Account of two spines with cervical ribs, one of which has a vertebra suppressed and absence of the anterior arch of the atlas. Journ. of anat. and phys. Vol.21. 1887.

9. Dwight, Th., Description of the human spines showing numerical variation in the Warren Museum of the Harvard Medical School. Anat. Anz. Bd.19. 1901.

10. Dwight, Th., The Skeleton. Piersol's human anatomy. Philadelphia and London. 1907.

11. Frizzi, E., Über die Wirbelsäule der Baining (Neu-Pommern). Zeitschr. f. Morph. u. Anthr. Bd.17. 1915.

12. Funke, E., Über einen Processus odontoideus atlantis hominis. Anat. Anz. Bd.14. 1898.

13. Hasebe, K., Die Wirbelsäule der Japaner. Zeitsehr. f. Morph. a. Anthr. Bd.15. 1913.

14. Hrdlička,A., Anomalous articulation and fusion of the atlas with the occipital bone. Washington Med. Annals. Vol. 3. No. 1. 1904.

15. Kubo, T., Untersuchungen über das Japanerskelett. Mitt. der Central japan. med. Gesell. Nr. 71. 1906. (Japaniseh)

16. Kollmann, J., Varianten am Os oecipitale besonders in der Umgebung des Foramen occipitale magnum. Anat. Anz. Bd. 30. 1907.

17. Krause, zitiert bei Hasebe.

18. Lehmann-Nitsche, R., Nouvelles recherches sur la formation pampéenne et l'homme fossile de la République argentine. Revista del Museo de la Plata. T. 14. 1907.

19. Poirier, P., Traite d'anatomie humaine. T. 1. Paris. 1899. 
20. Schnell, M., Über die Wirbelsäule des Menschen, des Gorilla und des Cercopithecus. Eine vergleichende osteologische Studie. Med. Diss. München (zitiert bei Lehmann-Nitsche.)

21. Swjetschnikow, Über die Assimilation des Atlas und die Manifestation des Occipitalwirbels beim Menschen. Arch. f. Anat. u. Phys. 1906.

22. Swjetschnikow, Über die Varianten des Occipitalwirbels. Anat. Anz. Bd. 32. 1908.

23. Tschugunow, S., Was ist Processus articularis atlantis Halbertsma s. Processus odontoideus atlantis hominis Funke? Nachrichten der Kais. Univ. Tomsk. Bd. 19. 1902. (Russisch)

24. Weigner, K., Über die Assimilation des Atlas und über die Variationen am Os occipitale beim Menschen. Anat. Hefte. Bd. 45. 1911.

25. Welker, Anthropologische Untersuchungen über das Atlantooccipitalgelenk bei Menschen und Affen. Dissertation. München. 1912.

26. Zanolli, V.,. Studi sullo atlante e sull'epistrofeos. Atti Acad. Sc. Ven.-Trent.-Istriana. Ser. 3. Anno 3. 1910.

27. Zoja, zitiert bei Zanolli.

\section{Erklärung der Abbildungen.}

Fig. 1. Vollständig geschlossenes Foramen atlantoideum laterale ist auf beiden Seiten bemerkbar. Natürliche Grösse von hinten gesehen.

Fig. 2. Auf der rechten und linken Seite ist unvollständiges Foramen atlantoideum posterius zu sehen. Natürliche Grösse, von oben etwas links: gesehen.

Fig. 3. Fast vollkommenes Foramen atlantoideum posterius ist wahrzunehmen. Natürliche Grösse, von rechts gesehen.

Ftg. 4. Ein Knochenvorsprung entspringt von dem hinteren Abschnitt der Massa lateralis und von der oberen Fläche des Arcus posterior. Natürliche Grösse, von oben etwas links gesehen.

Fig. 5. Der Atlas ist mit dem Condylus occipitalis fest verwachsen. Keine Rückbildung des Atlas bemerkbar. Auf ca. $\frac{1}{2}$ verkleinert photographiert, von unten gesehen.

Eig. 6. Der Atlas ist asymmetrisch ausgebildet, und zwar weicht er stark nach rechts ab. Natürliche Grösse, gerade von oben gesehen. 


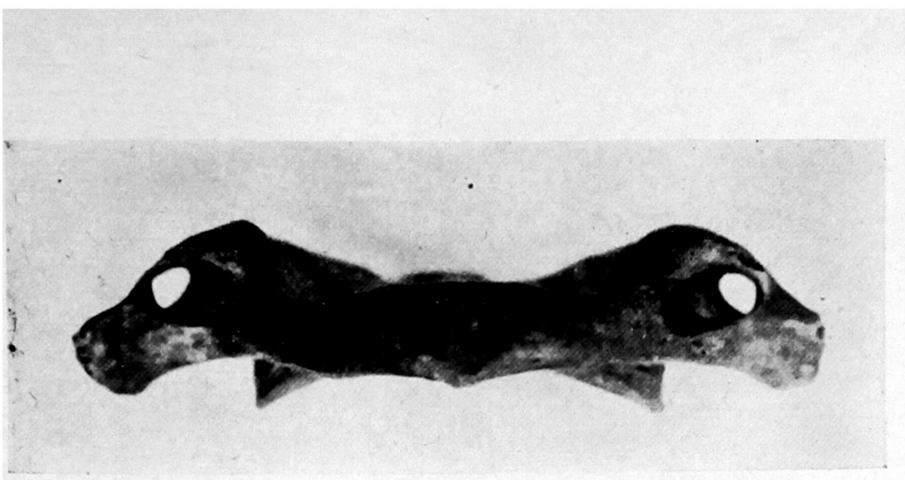

Fig. 1.

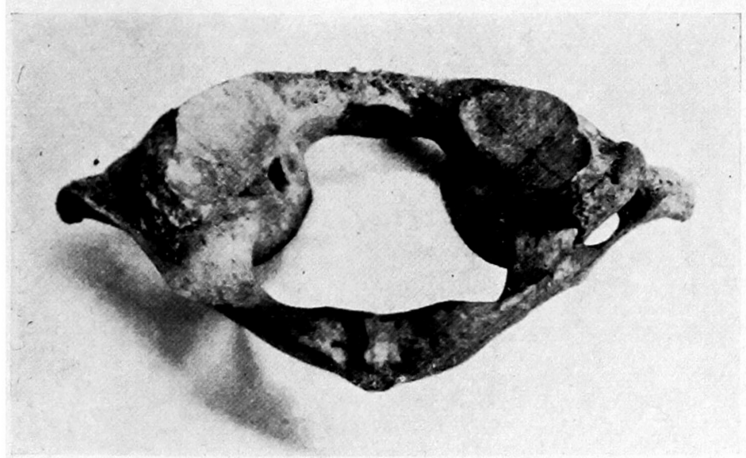

Fig. 2.

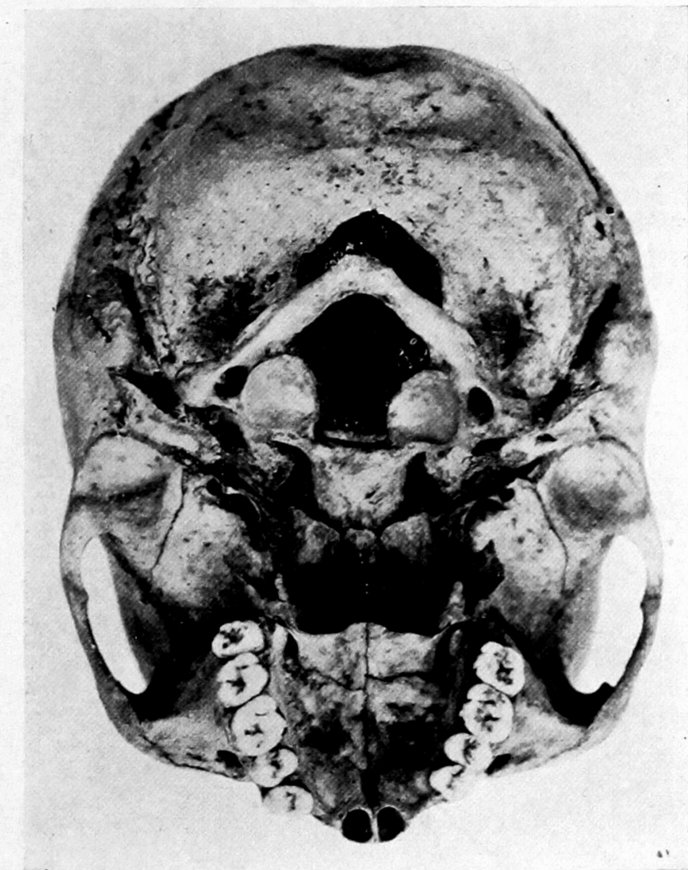

Fig. 5.

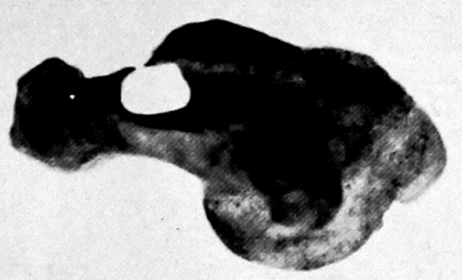

Fig. 3.

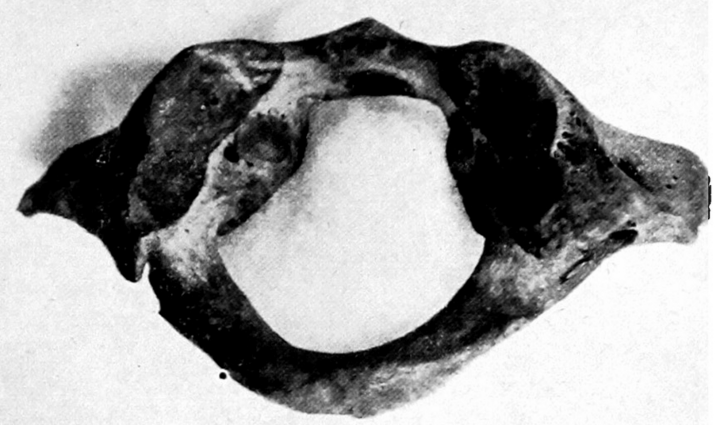

Fig. 4.

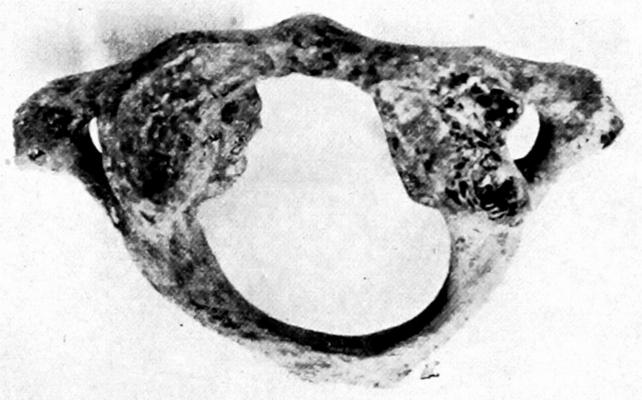

Fig. 6.

T. Tsusaki. 